\title{
Editorial introduction to the special issue on Uncertainty and Climate Change Adaptation
}

\author{
Tiago Capela Lourenço ${ }^{1}$ - Ana Rovisco ${ }^{1}$. \\ Suraje Dessai $^{2} \cdot$ Richard Moss $^{3} \cdot$ Arthur Petersen $^{4,5}$
}

Received: 22 April 2015 / Accepted: 20 May 2015 / Published online: 16 June 2015

(C) Springer Science+Business Media Dordrecht 2015

\section{Introduction}

Projections of future climate change, associated impacts and possible responses to cope with their effects are riddled with uncertainties, creating real and perceived barriers at all levels of policy- and decision-making. There is uncertainty about observed climate changes and their past effects on natural and human systems. There is uncertainty about the current state of the environment and its resilience to changes. There is even larger uncertainty about future changes in the climate system and their potential consequences on the environment and human societies. In many contexts, uncertainty is interpreted as a deficit of knowledge. However, in the absence of perfect knowledge, decisions are made every day, everywhere. Planning for climate change adaptation is a relatively new challenge, but decision-makers across sectors and scales are facing growing demand for adaptation-related decisions (such as strategies, measures and investments) that need to be made now or in the future. These are expected to protect human and natural systems against potential vulnerabilities or increase their resilience, while still allowing for sustainable development. This means taking into account an immense and

This article is part of a special issue on "Uncertainty and Climate Change Adaptation" with guest editors Tiago Capela Lourenço, Ana Rovisco, Suraje Dessai, Richard Moss, and Arthur Petersen.

Tiago Capela Lourenço

tcapela@fc.ul.pt

1 Faculty of Sciences, University of Lisbon, CE3C/CCIAM, Office 1.4.39, Campo Grande, 1749-016 Lisbon, Portugal

2 Sustainability Research Institute and ESRC Centre for Climate Change Economics and Policy, School of Earth and Environment, University of Leeds, Leeds LS2 9JT, UK

3 Joint Global Change Research Institute, 5825 University Research Court, Suite 3500, College Park, MD 20740, USA

4 Department of Science, Technology, Engineering and Public Policy (STEaPP), University College London, London, UK

5 Institute for Environmental Studies (IVM), VU University, Amsterdam, The Netherlands 
growing body of knowledge and data about climate and other global changes, including climate models and projections, socio-economic scenarios, impacts and vulnerability assessment tools, and adaptation appraisal techniques, among others. It also means coping with the wide range of (often disarming and technically complex) scientific concepts usually associated with climate change and adaptation research. Climate adaptation policy developments represent prime examples of decision-making practices that have to process and incorporate the array of uncertainties associated with scientific outcomes. In order to account for a wide spectrum of perspectives and to support better informed decisions, a growing and sometimes controversial body of knowledge about the climate system, potential changes and associated uncertainties needs to be communicated across disciplines and to those making decisions, in a clear and salient way. This is a great challenge for both researchers and decision-makers and represents the theme that underlines the development of this Special Issue in Climatic Change.

This Special Issue came together in the context of the CIRCLE-2 ERA-Net project (Climate Impact Research and Response Coordination for a Larger Europe - www.circle-era.eu) funded by the European Commission Seventh Framework Programme (EU FP7). The project ran between 2010 and 2014 and included the CIRCLE-2 Joint Initiative on Climate Uncertainties which gathered a variety of coordination and agenda setting activities under the theme of dealing with and communicating uncertainties in climate adaptation.

The development of this Special Issue involved a series of steps, namely:

1. An open call for abstracts submitted to a 2-day workshop on Uncertainty and Climate Change Adaptation, held at the Faculty of Sciences-University of Lisbon, Portugal, on 89 November 2012; a total of 72 abstracts were received of which 26 were selected to be developed into working papers, presented and discussed during the workshop;

2. Selection, by the scientific committee of the workshop, of the 12 most promising papers presented. The authors of these selected papers were then invited to submit a more advanced version;

3. Peer review of the 9 submitted papers, resulting in 7 papers being published in this Special Issue.

The primary objective of this Special Issue is to promote a timely discussion and valuable contribution of scientific insights on the issue of dealing with and communicating uncertainties in support of climate change adaptation. One key question that was posed at the start of the process binds the papers together:

- How do decision-making processes on climate change adaptation, at the multi-decadal timescale, envision the future and deal with related uncertainties?

Discussions during the preparation phase, and later between the workshop participants, revealed a set of additional queries, which were put forward as a departure point for further investigation:

- Which particular types or combinations of uncertainties (e.g. climatic, environmental, socioeconomic) matter most for those participating in adaptation planning and related decision-making processes?

- How can uncertainties be characterised and/or communicated to effectively inform climate adaptation decision-making processes? 
- How is scientific information in general and uncertainty in particular, commonly used or incorporated into decision-making practices, in the broad variety of sectors presently looking at climate adaptation?

- How effective have these approaches been in helping decision-makers and/or practitioners, and how relevant are they to the overall challenge of climate adaptation?

The papers of this Special Issue address these questions and provide new insights into the state-of-the-art of different fields related to climate change adaptation, in particular those dealing with the complex needs involving decision-making under uncertainty. Firstly, Kwakkel et al. (2015) illustrate the effect of using a model-driven approach in the development of robust adaptation strategies in the water management sector. By supporting the development of dynamic adaptive policies that can be changed over time, the authors seek to demonstrate how adaptation pathways allow for informed (and adaptive) decision-making under changing and uncertain environments. A paper by Wachsmuth (2015) provides empirical support to the importance of stakeholder integration in scenario-building exercises aiming at cross-sectoral regional adaptation. The author concludes that, in order to increase regional resilience, both climate and socioeconomic uncertainties have to be framed together with decision-makers if cross-sector fertilization is to become a reality. Watkiss et al. (2015) critically review some of the most commonly used decision support tools available for the economic appraisal of adaptation. The authors remark that while decision-making under uncertainty gains prominence in adaptation assessments, economic appraisal still relies heavily in deterministic approaches. By identifying key differences between used techniques, they conclude that it should be the type of adaptation problem and objectives shaping the suitability and use of different economic valuation tools. By exploring scenario and model uncertainty in cross-sectoral climate impact assessments, Dunford et al. (2015) evaluate cross-sectoral interactions and feedbacks, under a range of future scenarios that aim at supporting stakeholder dialogues. The authors conclude that addressing uncertainties using a mixed-method approach via the combination of formal numerical techniques, modeller interviews and network analysis provides considerable advantages over traditional validation-based assessments. Zegwaard et al. (2015) look at how knowledge is produced and incorporated into flood and water management and climate adaptation decision-making in the Netherlands. The authors apply an ontological politics approach to study how complexity, uncertainty and ignorance have been dealt with and incorporated into national policy-making and demonstrate how climate change has modified the way decisions are made and communicated. A paper by Gottschick (2015) contributes to the analysis and interpretation of how stakeholders handle uncertain climate change knowledge by framing discourses around four conceptual typologies: rational, no-regret, blissful and formative. The author uses empirical data to conclude that in most cases, uncertainty is not judged as problematic and uses the proposed framework to assess and elaborate on why this happens. Finally, Daron (2015) outlines some of the key challenges of using robust decision-making approaches to guide adaptation practice in developing countries. The author argues that the required combination of quantitative data with a qualitative understanding of competing environmental, socioeconomic and political factors data poses significant obstacles to the use of that approach in developing countries. He further concludes that this approach may well still be currently unattractive to decision-makers that are wary of diverting resources from areas seen as having more pressing needs, such as infrastructure and development issues. 
Acknowledgments This Special Issue was developed under the framework of the CIRCLE-2 ERA-Net project (funded by the European Commission Seventh Framework Programme under Grant Agreement No. 249685; www.circle-era.eu). The CIRCLE-2 Joint Initiative on Climate Uncertainties was generously funded by the Calouste Gulbenkian Foundation, Portugal. Suraje Dessai is supported by the European Research Council under the 7th Framework Programme (FP7/2007-2013)/ERC Grant Agreement No. 284369 and by the UK Economic and Social Research Council (ESRC) for the Centre for Climate Change Economics and Policy (CCCEP).

We would like to thank all the authors who have accepted our invitation to contribute to this Special Issue, the workshops participants and organisers and the FCUL team (Ana Gomes, Ângela Antunes and David Avelar) for their substantial support, as well as the reviewers for their comprehensive and valuable comments to these studies. We gratefully acknowledge the continuous assistance of Kristin Kuntz-Duriseti at Climatic Change who responded to our queries in such a professional and efficient manner.

Guest Editors.

\section{References}

Daron J (2015) Challenges in using a robust decision making approach to guide climate change adaptation in South Africa. Clim Chang. doi:10.1007/s10584-014-1242-9

Dunford R, Harrison PA, Rounsevell MDA (2015) Exploring scenario and model uncertainty in cross-sectoral integrated assessment approaches to climate change impacts. Clim Chang. doi:10.1007/s10584-014-1211-3

Gottschick M (2015) How stakeholders handle uncertainty in a local climate adaptation governance network. Clim Chang. doi:10.1007/s10584-014-1203-3

Kwakkel JH, Haasnoot M, Walker WE (2015) Developing dynamic adaptive policy pathways: a computerassisted approach for developing adaptive strategies for a deeply uncertain world. Clim Chang. doi:10.1007/ s10584-014-1210-4

Wachsmuth $\mathrm{J}$ (2015) Cross-sectoral integration in regional adaptation to climate change via participatory scenario development. Clim Chang. doi:10.1007/s10584-014-1231-z

Watkiss P, Hunt A, Blyth W, Dyszynski J (2015) The use of new economic decision support tools for adaptation assessment: a review of methods and applications, towards guidance on applicability. Clim Chang. doi:10. 1007/s10584-014-1250-9

Zegwaard A, Petersen AC, Wester P (2015) Climate change and ontological politics in the Dutch Delta. Clim Chang. doi:10.1007/s10584-014-1259-0 